\title{
Out-of-hospital cardiac arrest
}

\author{
Martin Porzer ${ }^{\mathrm{a}, \mathrm{b}}$, Eva Mrazkovaa ${ }^{\mathrm{a}}$, Miroslav Homza ${ }^{\mathrm{b}}$, Vladimir Janout ${ }^{\mathrm{a}}$
}

\begin{abstract}
Out-of-hospital cardiac arrest (OHCA) is a leading cause of death in developed industrial countries. The global worldwide average of OHCA incidence in adults is 95.9/100,000/year. European incidences vary according to source from 16 to $119 / 100,000 /$ year.

The aim of this study was to provide an overview of current information on OHCA. The incidences in various populations are discussed, along with the factors affecting the prognosis and outcome of these patients. The etiology and pathophysiological mechanisms are also described, especially in relation to the most common causes - acute and chronic forms of coronary artery disease and cardiomyopathies. Measures that could improve survival rates are discussed, with emphasis on the role of the general public and deployment of automatic external defibrillators.
\end{abstract}

Key words: cardiac arrest, sudden cardiac death, out of hospital cardiac arrest, epidemiology

Received: May 1, 2017; Accepted with revision: November 30, 2017; Available online: December 13, 2017 https://doi.org/10.5507/bp.2017.054

${ }^{a}$ Department of Epidemiology and Public Health, Faculty of Medicine, University of Ostrava, Czech Republic ${ }^{b}$ Department of Cardiovascular Diseases, University Hospital Ostrava, Czech Republic Corresponding author: Martin Porzer, e-mail:martin.porzer@gmail.com

\section{INTRODUCTION}

Out-of-Hospital Cardiac Arrest (OHCA) is a sudden malfunction of the cardiovascular system occurring outside a hospital environment. Such malfunction leads to a sudden decrease in perfusion of tissues including the central nervous system (particularly the brain's grey matter). Causes of the cardiac arrest are either primary (i.e. the cardiac arrest is caused by the heart's malfunction), or secondary (resulting from a respiratory arrest and anoxia, trauma, neurological, etc.). Prompt intervention - cardiopulmonary resuscitation (CPR) and removal of the cause - is necessary to prevent death ${ }^{1}$.

The definition of OHCA is somewhat ambiguous. It can be understood as any event of cardiac arrest out of hospital (resulting in death or not), which is however in essence an unquantifiable indicator. The cited papers therefore usually work with patients in whom resuscitation efforts were attempted, i.e., patients with cardiac arrest and attendance of the units of emergency medical system (EMS) $\left(\right.$ ref. $\left.^{2}\right)$.

OHCA is closely related to "sudden cardiac death" (SCD), which is defined as "unexpected death due to cardiac causes that occurs in a short time period (generally within $1 \mathrm{~h}$ of onset of symptoms)" (ref. ${ }^{1,3,4}$ ). Knowing that the majority of the OHCAs are due to primary cardiac causes (see below), the terms OHCA and SCD are very close, though not interchangeable. The principal difference lies in the fact that OHCA does not necessarily result in death.

\section{Epidemiology}

OHCA is a leading cause of death in developed industrial countries ${ }^{2,5}$. In the USA, it is among the top 6 causes of death ${ }^{6}$. Approximately 300,000 OHCA patients are treated annually in North America; around 275,000 in Europe ${ }^{2}$. The incidence of patients undergoing resuscitation as a result of cardiac arrest in Europe however ranges from 16 to $119 / 100,000$ inhabitants per year in various sources ${ }^{2,3,7}$.

The principal breakthrough in treatment of patients with in-hospital cardiac arrests came with creating specialized hospital units for treatment of acute coronary syndromes as the most frequent sources of cardiac arrest. The in-hospital mortality of these patients dropped from $30-35 \%$ to $15-17 \%$ during the 1960 s. However, the mortality of patients with acute coronary syndrome prior to admission to hospital has remained almost unchanged in the long-term, forming a significant statistical share of OHCA mortality ${ }^{8}$

The major analysis by Berdowski et al. (2010) aggregated 67 prospective papers dealing with OHCA incidence (1989-2009). 30 papers were from Europe, 24 from North America, 7 from Asia, and 6 from Australia. In total, a population of 100 million and 178,440 OHCA cases were systematic analysed. The global average OHCA incidence in the adult population was 95.9 per 100,000 inhabitants per year. The OHCA incidence was lower in Asia (52.5) than in Europe (86.4), North America (98.1), and Australia (112.9) (ref. $^{2}$ ).

OHCA is more common in men than in women ${ }^{1,2,3,9,10}$. The majority of OHCAs occur at home (more than 66\%), followed by workplaces and public places (approx. 20\%) or other unspecified locations ${ }^{11}$. The mean age of OHCA patients also differs between sexes ( $68 \pm 14$ years in males, $76 \pm 12$ years in females). Overall, the average age of patients in most studies is usually in the $7^{\text {th }}$ or $8^{\text {th }}$ decade (UK - 71 years, Sweden - 70 years, etc.). Men are significantly younger $(68 \pm 14$ years, respectively, $76 \pm 12$ years) (ref. $\left.{ }^{2,11,12}\right)$. 


\section{Etiology}

OHCAs may be caused by primary cardiac pathologies or not. In general, a majority of OHCAs are due to primary cardiac pathology with the cardiac arrest being usually caused either by a coronary artery disease or by dilated cardiomyopathy (together more than $90 \%$ of arrests) (ref. ${ }^{1,2,11-16}$ ). According to Berdowski, the global incidence of cardiac arrest with assumed primary cardiac cause is $55 / 100,000$ inhabitants ${ }^{2}$.

The causes of cardiac arrest are diagnosed in surviving patients through clinical and paraclinical tests during hospitalization. In patients who die of OHCA, however, the causes are not always determined as autopsy is often not performed for legal reasons. In many countries, autopsy is only performed if the cause of death is unknown, i.e. if not diagnosed by the examining physician ("acute myocardial infarction, cardiac arrest") (ref. $\left.{ }^{17}\right)$. Such a diagnosis is however declared by the attending physician in an overwhelming majority of cases. For example, as recorded in one German register, the units of medical emergency systems executed 569 missions to non-trauma patients showing no signs of life, 554 (97\%) of which were assumed to be death due to cardiac arrest. The remaining 15 patients were diagnosed with large devastating strokes (5 patients; $0.8 \%)$, primary respiratory arrest $(5 ; 0.8 \%), 2$ died of drowning $(2 ; 0.3 \%), 2$ of cardiac arrest caused by intoxication $(2 ; 0.3 \%)$, and one intervention dealt with a patient with terminal malign disease $(1 ; 0.17 \%)\left(\right.$ ref. $\left.^{11}\right)$.

Another paper based on a voluntary Swedish register comprised 14,065 patients (including paediatric patients) with cardiac arrest. In $67.1 \%$ of patients, the cardiac arrest was assumed to be of primary cardiac causes. Similarly to the set described above, clearly defined non-primary cardiac causes were recorded only in a small percentage of patients (suffocation $1 \%$, SIDS $0.8 \%$, drowning $1 \%$, suicide $1.1 \%$, primary pulmonary disease $2.9 \%$, trauma $1.7 \%$, drug overdose $1.5 \%$ - in total $10 \%$ ). The remaining $22.9 \%$ were classified as "cause unknown" or "other". The authors themselves assume that the majority of such unknown causes may also be cardiac. We can thus anticipate that primary cardiac pathology is the cause for $90 \%$ of patients with cardiac arrest ${ }^{12}$.

As mentioned above, a majority of primary cardiac causes of circulatory arrest are associated with coronary artery disease or dilated cardiomyopathy (more than 90\%) (ref. ${ }^{1}$ ) with coronary disease being the cause in $75-80 \%$. Cardiomyopathies, especially dilating cardiomyopathy, account for $10-15 \%$ of the primary cardiac causes of circulatory arrest. Only $5-10 \%$ of primary cardiac arrests occur forother reasons, including other forms of cardiomyopathies (in particular hypertrophic cardiomyopathy and arrhythmogenic right ventricular cardiomyopathy), primary failure of electrical conduction of the heart primary arrhythmogenic causes (primary ventricular fibrillation, failure of the electrical conduction system of the heart, long QT syndrome, Brugada syndrome, catecholaminergic polymorphic ventricular tachycardia, WPW syndrome, etc.), mechanical causes (aortic stenosis, massive pulmonary embolism), and others (fulminant myocarditis, etc.) (ref. $\left.{ }^{1}\right)$.
The age distribution of patients with sudden circulatory arrest is not surprising. Coronary disease is prevalent from the $5^{\text {th }}$ decade of life onwards. Primary cardiac arrests in younger patients (under 40) however show a different distribution of causes. In the USA, a group of 6.3 million military recruits (age $18-35$ ) was subject to a 25-year-long survey. Over that time, 126 sudden nontraumatic deaths occurred and all deceased were subject to an autopsy. The most frequent recorded cause was cardiac vein anomalies (33\%), followed by myocarditis $(20 \%)$. Coronary disease ranked third (16\%), followed by hypertrophic cardiomyopathy, biscupid aortic valve stenosis, and others ${ }^{13}$.

\section{Pathophysiology}

The key moment of circulatory arrest is the loss of heart's mechanical function and total loss of cardiac output as well as tissue perfusion. Immediately, in a matter of seconds, loss of consciousness and respiratory arrest follow.

The pathophysiology of circulatory arrest depends on the cause. Primarily non-cardiac causes originally lead to hypoxia and anoxia and subsequently to cardiac arrest. This occurs during suffocation, drowning, or during terminal stages of a primary pulmonary disease.

The mechanisms of the arrest of cardiac function include ventricular tachyarrhythmia, asystole, or pulseless electrical activity of the heart, which can be simultaneously detected by electrocardiography ${ }^{1}$. Ventricular tachyarrhythmias resulting in cardiac arrest include ventricular fibrillation and certain types of ventricular tachycardia (while other types of ventricular tachycardia can preserve blood circulation) (ref. ${ }^{1,3,18}$ ).

Pulseless electrical activity is an electrical activity of the heart which does not lead to a mechanical response of the heart as a pump and, therefore, to generation of peripheral pulse and organ perfusion. It is often caused by an obstacle in blood flow (massive pulmonary embolism, cardiac tamponade) or by primary respiratory failure. A long-term circulatory arrest with ventricular fibrillation as a primary rhythm results in development of asystole ${ }^{3}$.

In the case of acute myocardial infarction, different pathophysiological mechanisms arise, depending on the time elapsing from the coronary artery obstruction. In the first stage (within min of the arterial occlusion), changes in membrane potential occur, along with changes in intracellular transport of calcium. The intracellular concentration of potassium increases. The refractory period of the depolarized myocardium is not homogeneous, which results in an environment enabling micro re-entry circuits. These subsequently form a pathophysiological basis for the emergence of ventricular tachycardia and ventricular fibrillation. In the second stage, approx. after ten min, the mechanism of the so-called abnormal automaticity is activated and triggers actions related to the release of catecholamines ${ }^{4}$. Therapeutic intervention can prevent the impact of these processes and the emergence of malign arrhythmia in the acute stage of infarction. After $48 \mathrm{~h}$, scar tissue begins to develop and on the borders between the scars and healthy myocardium, macro re-entry circuits 
may be initiated, leading in turn to ventricular tachycardia, significantly affecting the patient's prognosis ${ }^{3}$. These facts must be taken into account for successful instrumental secondary prevention of malign arrhythmias in patients with ventricular arrhythmia induced by myocardial infarction ${ }^{19}$.

Delayed ventricular tachycardia can arise a long time after the myocardial infarction (months or years) through a different mechanism, with the already formed scar tissue acting as the environment for the development of macro re-entry circuits. Monomorphic ventricular tachycardia develops through a delayed activation of the peripheral part of the scar, in a heterogeneous tissue formed by viable myocardium and scar fibroblasts ${ }^{3,18}$.

If dilatory cardiomyopathy occurs, the left ventricle is macroscopically dilated and dysfunctional. Histological changes are non-specific and include degeneration of myofibrils and dispersed interstitial fibrosis. Intramyocardial re-entry is then the principal mechanism of the occurrence of ventricular tachycardia ${ }^{18}$.

Bradyarrhythmias, caused by a malfunction in formation or conduction of the impulses, are a less frequent pathophysiological agent of a sudden cardiac arrest / sudden cardiac death. Complete AV blockades, for which the symptoms can vary from dyspnoea, weakness, angina pectoris, CNS malfunction, or syncope up to sudden death, arise usually as a secondary problem in patients with coronary artery disease. Asystole leading to OHCA/SCD is caused either by a degeneration of the sinoatrial node without engaging a secondary pacemaker centre, or, more frequently, as a secondary effect of suppression of pacemaker centres (both the sinoatrial node and secondary centres) due to metabolic reasons. During a protracted hypoxemia (in terminal stages of various diseases or as a result of unsuccessful cardiopulmonary resuscitation), cellular hypoxia and acidosis lead to an electrolyte imbalance on both sides of cellular membranes. This in turn results in accumulation of extracellular potassium and in inhibition of the physiological function of primary and secondary pacemakers ${ }^{3,18}$.

\section{Initial rhythm}

An initial rhythm is the first rhythm recorded in patients with circulatory arrest, and is crucial for the prognosis of resuscitated patients.

Studies involving patients who had a Holter ECG monitor attached at the moment of their sudden death revealed interesting data. In a study including 157 such patients, the most frequent cause of death was ventricular tachycardia (83\%). The most prominent mechanism was ventricular fibrillation resulting from untreated ventricular tachycardia (62\%). The frequency of primary ventricular fibrillation was significantly lower $(8 \%)$. The remaining primary rhythms included torsade de pointes polymorphic ventricular tachycardia (13\%) and bradyarrhythmia (17\%) $\left(\right.$ ref. $\left.^{20}\right)$.

As suggested by studies focused on this topic ${ }^{2,3,6,12}$, it is necessary to distinguish between initial rhythm in rare cases of OHCA patients monitored at the time of the event, e.g. using Holter monitor and initial rhythm in typical pa- tients with OHCA (i.e. first rhythm recorded upon arrival of EMS units). In the latter group, the first documented rhythm is often asystole, which may be a consequence of a long-term circulatory arrest caused originally by a ventricular tachycardia or ventricular fibrillation (hereinafter $\mathrm{VT} / \mathrm{VF}$ ). It can be therefore expected that the representation of patients in whom the initial recorded rhythm is a shockable one in the latter group is lower than in the former. This can be however only interpreted as a result of time elapsed from the cardiac arrest till the beginning of monitoring.

According to the Berdowski's extensive meta-analysis in almost 179,000 OHCAs, ventricular fibrillation was the first recorded rhythm in $27 \%$ of patients. Europe and North America showed a higher frequency of ventricular fibrillation (35.5\%, respectively $30.4 \%$ ) compared to Asia (7.4\%). Taking into account the described assumption that VT/VF are responsible for a majority of circulatory arrests, we can hypothesize that the low frequency of VT/ $\mathrm{VF}$ in Asian registers is caused by a prolonged duration of the arrest at the moment of recording the initial rhythm (and thus a higher percentage of secondary asystole) (ref. ${ }^{2}$ ).

In a Swedish register $(n=14,065)$, the first documented rhythm was VT/VF in $43.3 \%$ of cases. In general, VT/VF as the initial rhythm was more commonly recorded in "witnessed cardiac arrests" than in "unwitnessed cardiac arrests" (ref. $\left.{ }^{10}\right)$. In a register collecting data over 18 years in Rochester, USA, 75\% of patients whose cardiac arrest was witnessed, had a shockable rhythm, unlike only $38 \%$ of patients with unwitnessed cardiac arrest. Both these results can be explained by the shorter period from the onset of symptoms to obtaining the first ECG curve for "witnessed cardiac arrests" (ref. $\left.{ }^{6}\right)$.

\section{Clinical picture}

As a result of the sudden loss of efficient circulation, failure in organ perfusion occurs. The central nervous system is the most sensitive to hypoxemia, which explains the obvious symptoms of circulatory arrest. The patient loses consciousness and postural tonus, and collapses. A tonic convulsive activity is a possible early symptom, which subsequently rapidly subsides. In patients after a short circulatory arrest with a spontaneous recovery most frequently due to an asystolic pause caused by a sinus node dysfunction - the convulsions can lead to a misinterpretation of the situation as an epileptic seizure.

At the beginning of the brain hypoxia/anoxia, a residual, pathological respiratory activity (conditioned by brainstem reflexes) can be observed - the so-called gasping/agonal respiration. Such inadequate respiration does not lead to perfusion of organs, in particular due to the absence of efficient circulation. When gasping is observed by a layman, the observer can be assuming that the patient is breathing spontaneously and therefore not recognize a circulatory arrest. During telephone contact with an emergency system, the operator should therefore put a major emphasis on questioning the character and rhythm of the patient's respiration. 
Müller et al. focused in their study aptly titled "How sudden is sudden cardiac death" in particular on the personal history and symptoms of OHCA patients $(n=406)$. Personal history was available for $352(87 \%)$ patients. 106 of those $(30 \%)$ had a coronary atherosclerotic disease confirmed by previous angiography. 16 patients experienced an episode of cardiac arrest and resuscitation in the past. 127 patients (36\%) had typical angina pectoris symptoms or were taking medication for it. It can be thus stated that two thirds of patients in the register had a clear evidence of the presence of coronary artery disease. Data on symptoms were collected for $232(80 \%)$ patients by the authors. Chest pain was present in 88 (22\%) patients; median duration was $120 \mathrm{~min}$, dyspnoea was present in 61 patients with median duration of 30 min. Remaining symptoms were considered a prominent symptom only in less than $10 \%$ of cases (nausea, vomiting, previous syncope, etc.). In $90 \%$ of cases, the duration of at least some of the symptoms was more than 5 min and the arrest occurred in less than 2 min from onset of any symptoms in 5 patients only ${ }^{11}$. The above documented register clarifies that the "sudden cardiac arrest" does not usually occur so unexpectedly as the title suggests.

\section{Prognosis / survival of OHCA patients}

The prognosis of a patient after a successful resuscitation depends largely on the condition of the central nervous system. Even a few min of anoxia may cause irreversible damage to the brain, the damage depending on the duration of anoxia.

Resuscitation immediately following a circulatory arrest increases the chances of patient's survival three times. Conversely, it has been proven that every minute without CPR reduces the chances of patient's survival by $10-15 \%$ (ref. $\left.{ }^{21}\right)$.

Average survival of adult OHCA patients is, according to a major global meta-analysis (2010), approx. 7\% (ref. ${ }^{2}$ ). A higher survival rate of $8-11 \%$ is reported in patients whose arrest occurred at a moment when EMS personnel was already on site (usually when the EMS was alerted already at the stage of prodromes) (ref. ${ }^{22}$ ).

The success rate of CPR and the subsequent treatment can be evaluated using several parameters, including patient survival to hospital admission, survival to discharge from hospital, one-month survival, one-year survival; a frequently discussed parameter is survival with favourable neurologic outcome.

The status of the central nervous system following successful resuscitation is obviously proportional to the return of spontaneous circulation (ROSC). In patients with initial recorded shockable rhythm (VT/VF), the probability of ROSC as well as the long-term survival is significantly higher than in patients with asystole or pulseless heart activity at the initial ECG record ${ }^{3,5,12}$.

The Berdowski analysis ${ }^{2}$ reports the average survival (to discharge from hospital) of adult OHCA patients as $5.6 \%$; in patients whose initial recorded rhythm was $\mathrm{VF}$, this rate was $11.4 \%$. As expected, the highest representation of ventricular fibrillation as the initial recorded rhythm (correlating with the highest percentage of patient survival to discharge) was found in registers from large urban agglomerations in developed countries (Amsterdam: 63\% of patients with VF, 43\% survival to discharge; Rochester: 56\% VF, 43\% survival; Katowice: $44 \%$ VF, $15 \%$ survival; etc.) (ref., ${ }^{2,6}$ ). In general, the highest OHCA survival rate is observed where EMS witnessed the arrest itself and the initial recorded rhythm was shockable $\mathrm{VT} / \mathrm{VF}$.

In the Swedish study in more than 14,000 patients, the reported average one month survival (after discharge from hospital) was 5\%. Where the initial recorded rhythm was $\mathrm{VT} / \mathrm{VF}$, that rate was $9.5 \%$, and even $12.7 \%$ in patients whose arrest was directly witnessed by EMS on site, which supports the findings mentioned above ${ }^{12}$.

It is necessary to realize that OHCA often affects people who are seriously ill beforehand. Besides the above, the prognosis is therefore also dependent on the initial condition of the patient, on the severity of the primary disease, associated illnesses, etc. For example, the outlook of an 80-year old patient with serious left ventricular dysfunction after repeated coronary episodes will be much poorer than that of a patient in $5^{\text {th }}$ decade of life with until now undamaged left ventricle.

\section{Care and prevention}

Recommendations for treatment of patients after a successful cardiopulmonary resuscitation are discussed in many studies, interpretation of which is beyond the scope of this paper. The latest recommendations are summarized in the European Resuscitation Council and European Society of Intensive Care Medicine Guidelines for Post-resuscitation Care (latest issue 2015) (ref. ${ }^{23}$ ).

As the majority of OHCAs are caused by the coronary atherosclerotic disease, the first step in treatment after a successful resuscitation is coronary angiography. Indication criteria for OHCA patients are identical to those in patients with sustained circulation - the principal criteria are clinical suspicion, ECG or lab results. The interpretation of the ECG curve however bears risk of incorrect assessment - the changes both in the ST segment and the left bundle branch block (LBBB) have a limited specificity in diagnosing the acute coronary syndrome in OHCA patients. According to Sideris et al., ECGs in 46\% of OHCA patients in whom angiography did not confirm coronary disease (105 patients) showed signs of acute coronary syndrome (ST elevation 16\%, ST depression $21 \%$, and LBBB $9 \%$ ) (ref. ${ }^{24}$ ). This can be explained by the effects of temporary anoxia in the myocardium during the cardiac arrest, which often manifests as ischemic changes on ECGs recorded immediately after ROSC. Another reason may lie in the pre-existent patient condition (LBBB for dilatory cardiomyopathy, ST depression in patients with excessive hypertrophy of left ventricle, etc.).

As the current level of professional care for OHCA patients is very high and there is not much room for improvement in that area (at least in developed countries), the efforts should probably be focused on measures leading to shortening the time to recovery of spontaneous circulation to minimum. 
The first of these measures is education of lay people including children in providing first aid and performing urgent basic resuscitation as well as in readiness to help. This can improve the proportion of OHCA patients in whom resuscitation has been attempted. Such education of lay people - potential patients - can also lead to an earlier identification of symptoms, thus reducing the risks of OHCA.

Preventive instrumental measures can be effective both at the level of an individual (implantable cardioverter defibrillator - ICDs) and of population (automatic external defibrillators - AEDs). Their principal benefit lies in facilitating prompt defibrillation.

At the individual level, this concerns patients who have already had a cardiac arrest with shockable rhythm in the past or patients with a significantly higher risk (in order of magnitudes) than the general population. The second group consists of patients with pre-existing cardiac disease and a high risk of SCD, such as, for example, patients after myocardial infarction with a serious depression of left ventricular systolic function, patients with dilatory cardiomyopathy with depression of left ventricular function, or patients with hypertrophic cardiomyopathy with family history of SCD (ref. ${ }^{19}$ ). A prevention ICD implantation is indicated in these patients. Such an implantation may be crucial for the patient, and, of course, also leads to an overall reduction in the incidence of VT/ VF OHCA (ref. $\left.{ }^{25}\right)$.

At the level of the population, the measures are based on deployment of automated external defibrillators (AEDs), thus increasing the probability of a very prompt defibrillation in places where the chance of OHCA occurrence is statistically high. Such locations are characteristic of a high concentration of people - stadiums, airports, etc. The growing utilization of AEDs combined with a higher ratio of VT/VF OHCA surviving patients has been reported in multiple studies ${ }^{5,26}$. A Dutch study from 2014 shows a threefold increase in frequency of AED utilization for OHCA resuscitation over a period of 7 years, and a statistically significant correlation with increase in number of patients discharged with a favourable neurological outcome ${ }^{5}$.

\section{CONCLUSION}

The progress in diagnostic and therapeutic methods has resulted in a significant, even decline in morbidity and mortality associated with many diseases. The milestones in the therapy of acute forms of ischemic heart disease were introduction of cardiac intensive care units, later followed by introducing methods for restoring perfusion in the area threatened by the blockage in the afflicted artery - initially the application of systemic thrombolysis, later the now preferred primary coronary angioplasty.

As a result of these improvements, the importance of the care for critically ill patients in the out-of-hospital environment is on the rise. The incidence of patients in whom resuscitation has been (successfully or unsuccessfully) attempted as a result of out-of-hospital cardiac arrest is not negligible ( 16 - 119/ 100,000/year) ( ref. $\left.^{7}\right)$. The mortality rate of such patients unfortunately remains high and can be targeted only with difficulty. It is important to mention that the survival of a patient does not necessarily mean a clinically favourable outcome as serious neurologic disabilities are frequent consequences of OHCA. Besides mortality, the term "survival with favourable neurologic outcome" is therefore another important parameter.

Due to the very close relationship between the period without spontaneous efficient circulation and the final neurologic outcome, it is obvious that for improving outcome of such patients, it is crucial to begin treatment as soon as possible after the cardiac arrest.

In countries with developed medicine and favourable geographical conditions, where EMS units capable of performing defibrillation can reach the place of the incident very quickly, a key role is played by the general public, or, more precisely, the patient's outlook is to a large extent affected by actions of laymen upon finding a person without signs of life. Of course, the patient himself/herself can significantly improve his chances for (neurologically favourable) survival by timely reporting any signs of a upcoming arrest - it is a well-known fact that in a majority of patients with $\mathrm{OHCA} / \mathrm{SCD}$, some of the prodromes have been clearly and relatively early expressed.

Besides educating the general public in life-saving actions, preventive implantation of cardioverters/defibrillators in high risk patients and deployment of AEDs in places with a high concentration of people are other methods for improving the outcome of OHCA patients.

\section{Search strategy and selection criteria}

The presented paper is focused on providing the most complex overview concerning the issue of OHCA. Reviewed were papers and expert reports from 1989 to 2015 dealing with the issues of sudden cardiac death and cardiopulmonary resuscitation. The rather old paper from 1989 was included due to its outstanding relevance and almost unrepeatable results. Papers were searched through PubMed database. Searched terms were "cardiac arrest", "acute coronary syndrome" and "sudden cardiac death". Due to the need of providing information about cardiac arrest pathophysiology comprehensive books were also cited. Only English language papers were reviewed.

\section{REFERENCES}

1. Hohnloser SH. Sudden Cardiac Death and Resuscitation. In: Camm AJ, Lüscher TF, Serryus PW. The ESC Textbook of Cardiovascular Medicine. Oxford: Blackwell Publishing; 2006, p. 973-991.

2. Berdowski J, Berg RA, Tijssen JG, Koster RW. Global incidences of out-of-hospital cardiac arrest and survival rates: systematic review of 67 prospective studies. Resuscitation 2010;81(11):1479-87. doi: 10.1016/j.resuscitation.2010.08.006

3. Pleskot M. Nahla srdecni smrt. In: Vojacek J., Kettner J. Klinicka kardiologie. Hradec Kralove: Nucleus HK; 2012. p. 880-902.

4. Rubart M, Zipes DP. Mechanisms of sudden cardiac death. J Clin Invest 2005;115(9):2305-15. doi: 10.1172/JCI26381

5. Blom MT. Improved Survival After Out-of-Hospital Cardiac Arrest and Use of Automated External Defibrillators - Clinical Perspective. Circulation 2014;130(21):1868-75. doi: 10.1161/ CIRCULATIONAHA.114.010905 
6. Agarwal DA, Hess EP, Atkinson EJ, White RD. Ventricular fibrillation in Rochester, Minnesota: experience over 18 years. Resuscitation 2009;80(11):1253-8. doi: 10.1016/j.resuscitation.2009.07.019

7. Gräsner JT, Herlitz J, Koster RW, Rosell-Ortiz F, Stamakis L, Bossaert L. Quality management in resuscitation-towards a European cardiac arrest registry (EuReCa). Resuscitation 2011;82(8):989-94. doi: 10.1016/j.resuscitation.2011.02.047

8. Riedl M. Dejiny kardiologie. Praha: Galén; 2009.

9. Franek O, Pokorna M, Sukupova P. Pre-hospital cardiac arrest in Prague, Czech Republic-The Utstein-style report. Resuscitation 2010;81(7):831-5. doi: 10.1016/j.resuscitation.2010.03.005

10. Pleskot $M$, et al. Characteristics and short-term survival of individuals with out-of-hospital cardiac arrests in the East Bohemian region. Resuscitation 2006; 68(2): 209-220. doi:10.1016/j.resuscitation.2005.06.017

11. Müller D, Agrawal R, Arntz HR. How sudden is sudden cardiac death? Circulation 2006; 114(11):1146-50. doi: 10.1161/ CIRCULATIONAHA.106.616318

12. Holmberg M, Holmberg S, Herlitz J, Gardelöv B. Survival after cardiac arrest outside hospital in Sweden. Resuscitation 1998;36(1): 29-36.

13. Atwood C, Eisenberg MS, Herlitz J, Rea TD. Incidence of EMS-treated out-of-hospital cardiac arrest in Europe. Resuscitation 2005;67(1):7580. doi: 10.1016/j.resuscitation.2005.03.021

14. Jacobs I, et al. Cardiac arrest and cardiopulmonary resuscitation outcome reports: update and simplification of the Utstein templates for resuscitation registries.: A statement for healthcare professionals from a task force of the international liaison committee on resuscitation. Resuscitation 2004;63(3):233-49. doi: 10.1161/01. CIR.0000147236.85306.15

15. Belohlavek J, Kucera K, Jarkovsky J, Franek O, Pokorna M, Danda J, Skripsky R, Kandrnal V, Balik M, Kunstyr J, Horak J, Smid O, Valasek J, Mrazek V, Schwarz Z, Linhart A. Hyperinvasive approach to out-of hospital cardiac arrest using mechanical chest compression device, prehospital intraarrest cooling, extracorporeal life support and early invasive assessment compared to standard of care. A randomized parallel groups comparative study proposal.."Prague OHCA study". J Transl Med 2012;10:163. doi: 10.1186/1479-5876-10-163

16. Zheng ZJ, Croft JB, Giles WH, Mensah GA. Sudden cardiac death in the United States, 1989 to 1998. Circulation 2001;104(18): 2158-63.

17. Zakon $89 / 2012$ Sb. Obcansky zakonik Ceske Republiky

18. Kautzner J. Komorové tachyarytmie. In: Vojacek J, Kettner J. Klinická kardiologie. Hradec Králové: Nucleus HK; 2012. p. 313-328.
19. Priori SG, Blomström-Lundqvist C, Mazzanti A, Blom N, Borggrefe M, Camm J, Elliott PM, Fitzsimons D, Hatala R, Hindricks G, Kirchhof P, Kjeldsen K, Kuck KH, Hernandez-Madrid A, Nikolaou N, Norekvål TM, Spaulding C, Van Veldhuisen DJ. 2015 ESC Guidelines for the management of patients with ventricular arrhythmias and the prevention of sudden cardiac death. Eur Heart J. 2015;36(41):2793-2867. doi: 10.1093/eurheart/ehv316

20. De Luna AB, Coumel P, Leclercq JF. Ambulatory sudden cardiac death: mechanisms of production of fatal arrhythmia on the basis of data from 157 cases. Am Heart J 1989;117(1):151-9.

21. Janota T. Kardiopulmonalni resuscitace. In: Vojacek J, Kettner J. Klinická kardiologie. Hradec Kralove: Nucleus HK; 2012. p. 871-879.

22. Sasson C, Rogers MA, Dahl J, Kellermann AL. Predictors of survival from out-of-hospital cardiac arrest. Circulation: Cardiovascular Quality and Outcomes 2010;3(1):63-81. doi: 10.1161/ CIRCOOUTCOMES.109.889576

23. Nolan JP, Soar J, Cariou A, Cronberg T, Moulaert VR, Deakin CD, Bottiger BW, Friberg H, Sunde K, Sandroni C; European Resuscitation Council; European Society of Intensive Care Medicine. European Resuscitation Council and European Society of Intensive Care Medicine Guidelines for Post-resuscitation Care 2015. Resuscitation 2015;41(12):2039-56. doi: 10.1007/s00134-015-4051-3.

24. Sideris G, Voicu S, Dillinger JG, Stratiev V, Logeart D, Broche C, Vivien B, Brun PY, Deye N, Capan D, Aout M, Megarbane B, Baud FJ, Henry $P$. Value of post-resuscitation electrocardiogram in the diagnosis of acute myocardial infarction in out-of-hospital cardiac arrest patients. Resuscitation 2011;82(9):1148-53. doi: 10.1016/j.resuscitation.2011.04.023

25. Hulleman M, Berdowski J, de Groot JR, van Dessel PF, Borleffs CJ, Blom MT, Bardai A, de Cock CC, Tan HL, Tijssen JG, Koster RW. Implantable Cardioverter defibrillators have reduced the incidence of resuscitation for out of hospital cardiac arrest caused by lethal arrhythmias. Circulation 2012;126(7):815-21. doi: CIRCULATIONAHA. 111.089425

26. Weisfeldt ML, Sitlani CM, Ornato JP, Rea T, Aufderheide TP, Davis D, Dreyer J, Hess EP, Jui J, Maloney J, Sopko G, Powell J, Nichol G, Morrison LJ; ROC Investigators.Survival after application of automatic external defibrillators before arrival of the emergency medical system: evaluation in the resuscitation outcomes consortium population of 21 million. J Am Coll Cardiol 2010;55(16):1713-20. doi: 10.1016/j.jacc.2009.11.077 